\title{
THE USE OF AUTOGENOUS BONE WITH AND WITHOUT PLATELET RICH FIBRIN AROUND IMMEDIATELY PLACED DENTAL IMPLANT
}

\author{
Abla I. Elkhawass ${ }^{1} B D S$, Magda M. Saleh ${ }^{2} P h D$, Saeeda M. Osman ${ }^{2} P h D$,
} ABSTRACT

INTRODUCTION The success of implant therapy depends primarily on appropriate treatment planning, case selection, properly performed implant placement surgery and good implant to bone adhesion. This can be achieved by several types of grafts around the implant.

OBJECTIVES: To evaluate clinically and radiographically the use of autogenous bone graft with and without platelet rich fibrin around immediately inserted implants and to evaluate implant stability by resonance frequency analysis device (Osstell).

MATERIALS AND METHODS: Clinical trial on 14 patients indicated for maxillary anterior teeth extraction selected from the outpatient clinic in oral and maxillofacial surgery department, Alexandria University were divided into two groups. In group A: the implant was inserted with autogenous bone collected from freshly extracted socket mixed with platelet rich fibrin as a filling of the gap, while in group B: the implant was inserted with autogenous bone collected from freshly extracted socket as filling of the gap.

RESULTS clinical and radiographical evaluation of the implants showed good osseointegration between the bone and the immediate implant in both groups.

CONCLUSIONS: The results show that the harvested autogenous bone may be useful when small amount of bone is required and that the PRF helps in bone formation and osseointegration in a short period of time.

KEYWORDS: Immediate implant, autogenous bone, Platelet rich fibrin.

1-B.D.S Oral and Maxillofacial Surgery Department, Faculty of Dentistry, Alexandria University, Alexandria, Egypt 2-Professor, Oral and Maxillofacial Surgery Department, Faculty of Dentistry, Alexandria University, Alexandria, Egypt

\section{INTRODUCTION}

The clinical replacement of lost teeth by ossteointegrated implants has been one of the most significant advances in dentistry (1). Immediate placement of a dental implant in an extraction socket may allow preservation of the socket and the surrounding jaw bone (2). Advantages of this treatment approach have been claimed to be reduction in the number of surgical interventions, shorter time treatment, an ideal three dimensional implant positioning, preservation of the alveolar ridge at the site of extraction and adequate soft tissue esthetics $(3,4)$.

Bone grafting has become one of the most common surgical techniques. An ideal bone graft must be nontoxic, non-carcinogenic, readily available, easy to use and mostly induces bone formation.(5) The grafting material will support the tissues surrounding the extraction site and by time will be replaced by new alveolar bone; this bone will be excellent support for future implant placement (6).

Autogenous bone is often referred to as the gold standard grafting material as it has osteoinductive, osteoconductive and osteogenic properties (7) .On the other hand, Platelet rich fibrin PRF is a second generation platelet concentrate widely used to accelerate soft and hard tissue healing (8). The natural concentrate of PRF is produced without any anticoagulants. (9) It is an autofocus fibrin matrix containing many kinds of growth factors and cytokines.(10) Growth factors are known to promote cell proliferation, cell differentiation, cell motility and matrix synthesis by binding to specific cell surface receptors. It is generally accepted that growth factors have an essential role in the healing process and tissue formation.(11)

In this study a combination of autogenous bone graft with and without PRF are used around the immediately placed dental implant following single rooted tooth extraction. Therefore, this study was to evaluate bone regeneration around implants grafted with autogenous bone combined with PRF in comparison with autogenous bone alone.

\section{MATERIALS AND METHODS \\ Patient selection}

A total of 14 patients, in good general health condition, were selected from the outpatient clinic in oral and maxillofacial surgery department, Alexandria University and were divided into two groups. Patient selection criteria for this study was established so as to include non- smoker patients having maxillary anterior teeth indicated for extraction with age ranging from 20-40 years with sufficient bone volume and good oral hygiene .While the exclusion criteria were presence of infection, insufficient inter-occlusal and mesiodistal spaces for implant, bruxism or clenching, current chemotherapy or radiotherapy, alcohol or drug abuse and the presence of systemic diseases.

The patients were divided into two groups. In group A (study group) the implant was inserted with autogenous bone collected from freshly extracted socket mixed with platelet rich fibrin as a filling of the gap, while in group $\mathrm{B}$ (control group) the implant was inserted with autogenous bone collected from freshly extracted socket alone.

An Ethical Committee evaluated and accepted the guidelines of the study. All patients gave their informed consent to participate in the study.

\section{Materials}

Dentium SuperLine Implant System (3105 Trade Tower 159, Samsung-dong, Gangnam-gu, Seoul, Korea.) was used in this study with the dentium surgical kit containing drills with different diameters, harvest bone drill to collect the bone, graduated depth gauge and paralleling pins. PRF was used as a graft material with the autogenous bone collected intraoperatively. Osstell mentor was used to measure the implant stability value. 


\section{Methodology \\ Preoperative phase}

Diagnosis was done preoperatively by X-ray examinations, including panoramic radiography and cone beam computed tomography to select an implant of adequate length.

All surgeries were performed under sterile conditions. Chlorhexidine $0.2 \%$ HCL (Hexitol mouth wash, the Arab drug Co., Cairo, Egypt) was used to rinse the oral cavity for 2 min prior to surgery.

\section{Operative phase}

Local anesthesia consists of Mepevacaine HCL 2\% (Mepevacaine L of Alexandria Co. for Pharmaceuticals, Alexandria, Egypt) and epinephrine as Levonordefrin $1: 200,000$ in a $1.8 \mathrm{ml}$ carpule was infiltrated into the maxillary surgical site. Teeth were carefully removed by using a gentle atraumatic extraction forceps in order to protect and preserve the alveolar bone for immediate implant insertion (Figure 1).

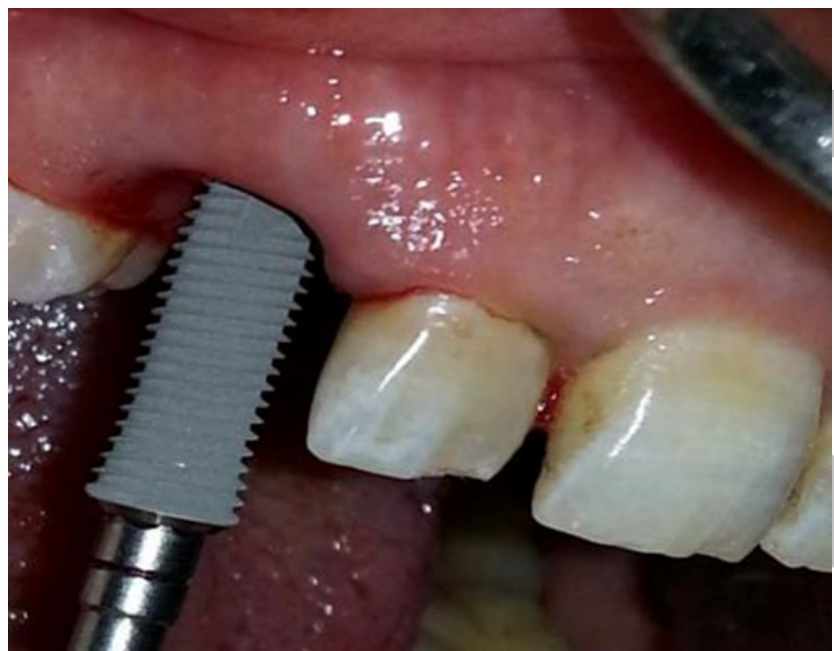

Figure 1: A photo that shows the insertion of the fixture in the empty socket after extraction in group A patient.

Sockets were curetted and irrigated with $0.9 \%$ normal saline to remove granulation tissue and residual periodontal ligament.

Drilling by Harvest bone drill, this process was carried out at low speed. The bone was collected directly from the drill. The threads of the harvest drill have a retentive design (deep grooves) that enables the storage of the cut trabecular bone.

Autograft preparation: After the drill was removed from the socket, a periotome or another instrument was used to extract the tissue from the harvest drill. The tissue was deposited in a small sterile dappen dish. The mixture was used to fill the gap between the socket and the titanium implants.

Preparation of platelet rich fibrin: by withdrawing 10 $\mathrm{ml}$ of venous blood from the antecubital fossa of the patient. Then the blood was transferred into $10 \mathrm{ml}$ sterile tube without anticoagulant and immediately the blood is centrifuged using tabletop centrifuge at $3000 \mathrm{rpm}$ for 10 minutes.

PRF (Fibrin clot) formed in between the acellular plasma on top and the red blood cells at the bottom, was separated using sterile tweezers and scissors.

Flapless placement of implant fixture was performed using a surgical motor at a low speed in the empty socket.
In group A the collected bone was mixed with platelet rich fibrin produced from the blood sample taken from the patient intra-operatively (Figure 2)

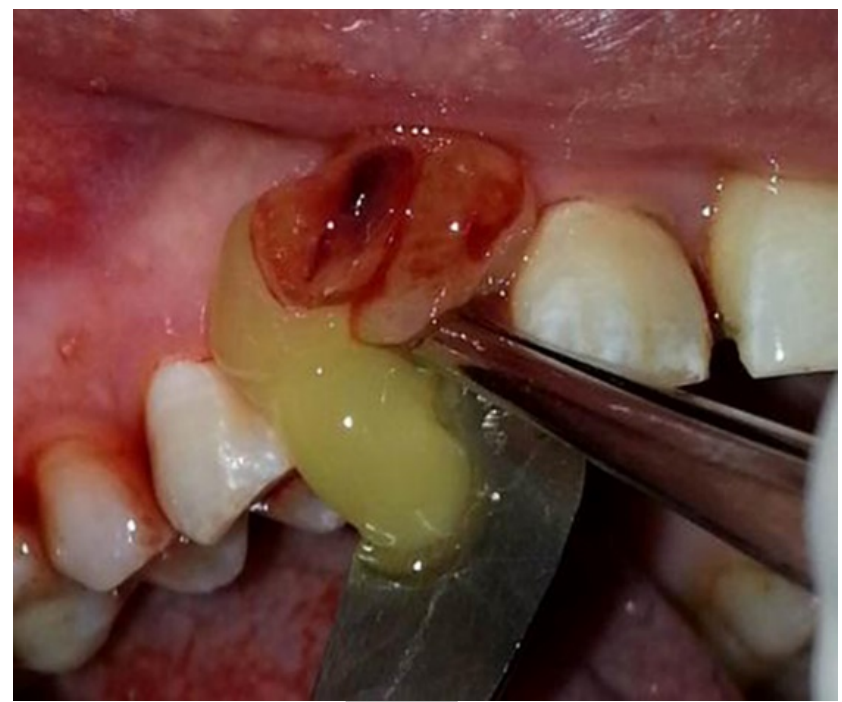

Figure 2: Placing the PRF and the autogenous bone around the implant in group A patient.

In group B the implant was placed immediately after root extraction (Figure 3).

Then the collected bone was grafted alone around the implant in the extraction socket (Figure 4).

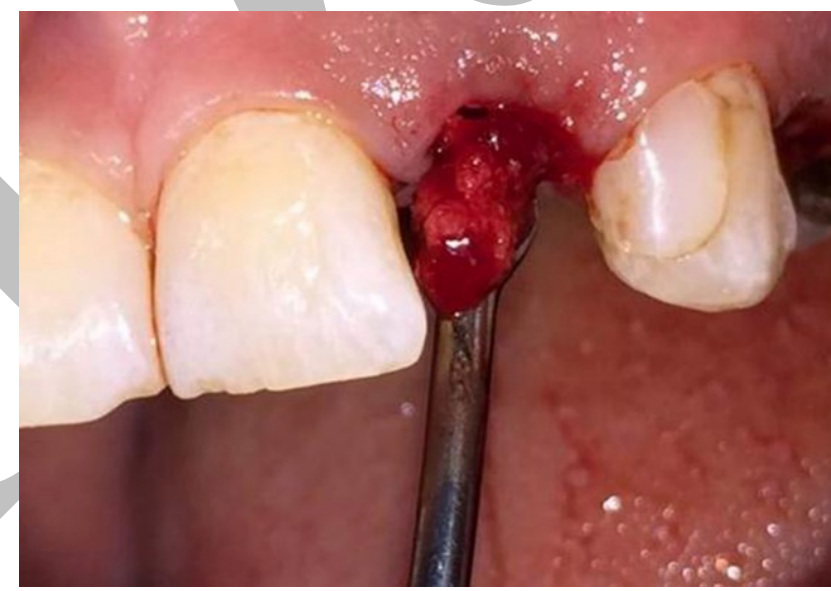

Figure 3: Implant placement in the empty socket after extraction in group $\mathrm{B}$ patient.

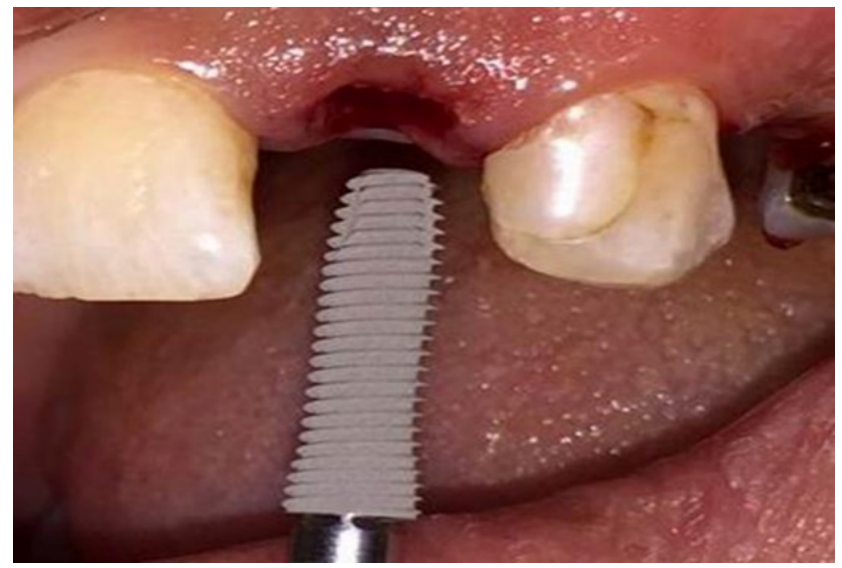

Figure 4: A photograph showing the harvested bone used as autogenous bone graft in group B patient 
The harvested material was used to fill the gap between the socket and the implant.

In both groups the smartpeg was screwed to the implant fixture, we used the Resonance Frequency Analysis Device (Osstell) holding its probe close to the smartpeg without touching it. The device beeps displaying the implant stability quotient (ISQ) on its screen.

The cover screw was screwed on the fixture and the wound was closed by $3 / 0$ black silk suture.

\section{Postoperative Phase}

Following surgery, patients were instructed not to brush or irritate the surgical sites for 10 days, to irrigate their mouth with chlorhexidine $0.2 \%$ HCL (Hexitol mouth wash, the Arab drug Co., Cairo, Egypt) 3 times a day for 1 week, and to maintain a soft diet for about 6 weeks. Non-steroidal antiinflammatory drugs (Diclofenac potassium 50mg tablets, Novartis Pharma AG., Basle, Switzerland) and antibiotics (Amoxicillin 875 mg+ clavulanic acid 125 mg, 1,000 mg, 2 times daily, Medical Union Pharmaceuticals, Abu Sultan, Ismailia, Egypt) were prescribed to be taken for 1 week.

\section{Follow up Phase}

One week after implant insertion, the sutures were removed. Regular clinical follow up was done every month to evaluate the pain, probing depth and modified sulcus bleeding index while radiographical follow up was done to measure the bone density throughout the 3 months healing period for both groups (Figure 5, 6).

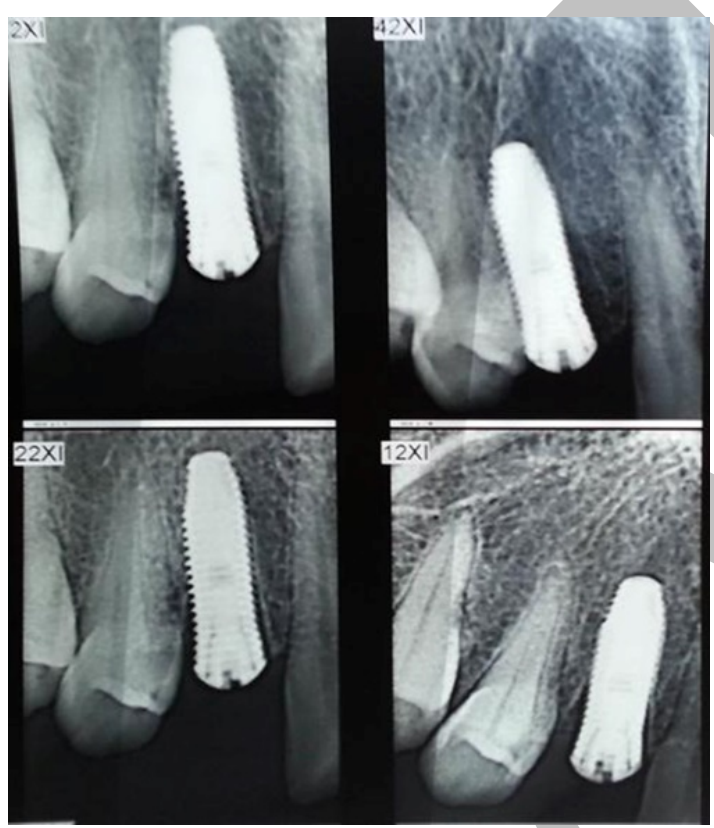

Figure 5: The X-ray for the site 3 months later in group A patient.

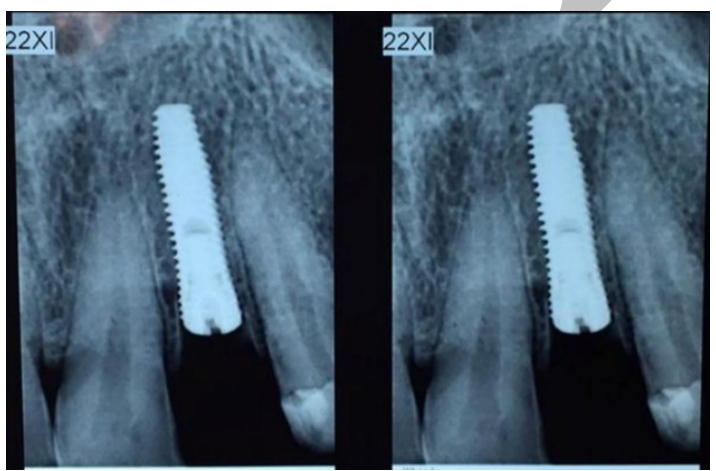

Figure 6: The Xray shows good bone formation around the implant in 3 months in group B patient.

\section{Prosthetic phase}

A provisional non-functional crown was done for all the patients during the healing period, and then a good shade selection was done for permanent porcelain fused to metal crown.

Statistical analysis of the data collected throughout the study period for both groups was done by Paired t-test and student t-test.

\section{RESULTS}

\section{Clinical results}

Fourteen patients (eight females and six males) having maxillary anterior single rooted teeth indicated for extraction were included in this study. Patients were divided into two groups; group $\mathrm{A}$ and group $\mathrm{B}$. Where seven patients (three females and four males) were assigned to group A (the study group), while the other seven (five females and two males) were assigned to group B (the control group).

Eight patients had badly destructed central incisors indicated for extraction. Four patients had lateral incisors as remaining roots indicated for extraction. Two patients had canines to be extracted. Fourteen implants were used with different length and diameters $(4.0 \mathrm{mmx} 12 \mathrm{~mm}$, 3.6mmx $10 \mathrm{~mm}$ and $4.0 \mathrm{mmx} 14 \mathrm{~mm}$ ).

Statistical analysis of the data collected showed that all patients experienced no pain to mild pain at the surgical site in the day of surgery.

Probing depth was measured in millimeter (mm) for all the axial surfaces of all implants. On the third month, the mean probing depth scores for the study group was $1.30 \mathrm{~mm}$ $\pm 0.54 \mathrm{~mm}$ with a minimum recorded value of $0.75 \mathrm{~mm}$ and a maximum recorded value of $2 \mathrm{~mm}$, while the mean probing depth scores for the control group was $2.10 \mathrm{~mm} \pm$ $0.29 \mathrm{~mm}$ with a minimum recorded value of $1.75 \mathrm{~mm}$ and a maximum recorded value of $2.5 \mathrm{~mm}$. None of the implants showed any signs of mobility.

Modified sulcus bleeding index was recorded according to Mombelli technique (12). The decrease in the modified sulcus bleeding index on the 3rd month was significant. ( $p$ $=0.010$ ).

Implant Stability Quotient ISQ or ISQ Scale, is a scale from 1 to 100 and is a measure of the stability of an implant.

The ISQ scale has a non-linear correlation to micro mobility. Many scientific studies have reported that high stability means $>70$ ISQ, between 60-69 is medium stability and $<60$ ISQ is considered as low stability. ISQ was measured in all patients using the resonance frequency analysis technique by the Osstell Tm device,. In group A was 77.71 \pm 1.60 ISQ, and in group B was 71.29 \pm 4.61 ISQ.

The increase in ISQ after 3rd month between the studied groups was statistically significant. $(p=0.009)$

\section{Radiographic results}

The bone density in group A was statistically significant throughout the follow up period from immediately postoperative (93.36 $\pm 17.50 \mathrm{HU})$ to $(120.39 \pm 18.04 \mathrm{HU})$ in the 3rd month, While in group B the bone density was statistically significant throughout the follow up period from immediately post-operative $(74.70 \pm 9.82 \mathrm{HU})$ to (118.50 $\pm 11.63 \mathrm{HU})$ in the 3rd month. 


\section{DISCUSSION}

This study was designed to evaluate bone regeneration following implant placement in immediate extraction socket using autogenous bone alone and autogenous bone combined with PRF.

A total of fourteen patients (eight females and six males) having maxillary anterior single rooted teeth indicated for extraction were included in this study. Their ages ranged between twenty and forty years with mean age of thirty years. Patients were divided into two groups; group A and group B. Where seven patients (three females and four males) were assigned to group A (the study group), while the other seven (five females and two males) were assigned to group B (the control group). All fourteen patients had undergone surgical procedures for immediate implant placement.

All patients in the current study were non-smokers. Nicotine, which is the major component of tobacco, is cytotoxic to the cells leads to vasoconstriction, decreased tissue perfusion and increased blood viscosity leading to microvascular occlusion and reduces alveolar blood supply, which compromise bone formation as reported in Kasat and Ladda study (13).

Bone regeneration using autogenous bone was selected in this study because it is referred as the gold standard grafting material as it has osteoinductive, osteoconductive and osteogenic properties; in addition it maintains bone structures such as minerals and collagen, as well as viable osteoblasts and Bone Morphogenic Proteins BMPs $(14,15)$. This is in coordinance with the findings of Santagata et al. (16), who harvested autologous bone graft during implant preparation and studied it histologically.

PRF was used as it is the latest development among the platelet concentrates. It is a second-generation platelet concentrate widely used to accelerate soft and hard tissue healing and is a strictly autologous fibrin matrix containing a large quantity of platelet and leukocyte cytokines (17). This agrees with the findings of El Kenawy et al. (18) and Boora et al. (19), who studied the effect of PRF around the immediate dental implant.

Several studies have reported that Resonance Frequency Analysis (RFA) via the Osstell ISQ system is a useful tool to analyze primary stability after implantation, as well as the degree of stability after osseointegration $(20,21)$. Osstell resonance frequency analysis device is a simple, noninvasive diagnostic device that many clinicians currently use, the RFA device provides a useful measurement to assess ossteointegration which is in coordinance with the findings of Qabbani et al. (22).

Although there was no difference between the study and control groups in accordance to peri-implant bone density, yet both groups showed increase in peri-implant bone density starting from the immediate postoperative period to the end of the 3 months of the evaluation period which indicates osseointegration of all implants.

\section{CONCLUSION}

The addition of PRF to autogenous bone graft accelerates the regenerative capacity of bone. They give a predictable clinical and radiographic evidence of bone formation and faster healing than using autogenous bone alone.

\section{CONFLICT OF INTEREST}

The authors declare that they have no conflicts of interest.

\section{REFERENCES}

1- Branemark PI, Zarb GA, Albrektsson T. Tissue-integrated prostheses: Osseointegration in clinical dentistry. 1985; 11-71.

2- Belser UB, Bernard D. Implant placement in the esthetic zone. In: Lindhe J, Karring T, Lang NP. Clinical periodontology and implant dentistry. 4th ed: Oxford, Berlin Paris: B Lackwell Munksguard publishing co; 2003; 915-9.

3- Sennerby L, Gottlow J. Clinical outcomes of immediate/early loading of dental implants. A literature review of recent controlled prospective clinical studies. Austl Dent J. 2008; 53: 82-8.

4- Chen ST, Wilson TG, Hammerle CH. Immediate or early placement of im-plants following tooth extraction: review of biologic basis, clinical procedures, and outcomes. Int J Oral Maxillofac Implants. 2004; 19:12-25.

5- Ashman A. Postextraction ridge preservation using synthetic alloplast. Implant Dent 2000; 9: 168-76.

6- Lee H, Choi B, Jung J, Zhu S, Lee S. Maxillary sinus floor augmentation using autogenous bone grafts and plateletenriched fibrin glue with simultaneous implant placement. Oral Surg Oral Med Oral Pathol Oral Radiol Endod. 2007; 103: 32933.

7- Soffer E, Ouhayoun JP, Anagnostou F. Fibrin sealants and platelet preparations in bone and periodontal healing. Oral Surg Oral Med Oral Pathol Oral Radiol Endod. 2003; 95: 521-8.

8- Lucarelli E, Beretta R, Dozza B, Tazzari P, O’Connell S, Ricci $\mathrm{F}$, et al. A recently developed bifacial platelet-rich fibrin matrix. Eur Cell Mater 2010; 20: 13-23.

9- Sunitha Raja V, Munirathnam Naid E. Platelet-rich fibrin: evolution of a second-generation platelet concentration. Indian J Dent Res 2008; 19: 42-6.

10-Kathuria A, Chaudhry S, Talwar S, Verma M. Endodontic management of single rooted immature mandibular second molar with single canal using MTA and platelet rich fibrin membrane barrier: A case report J Clin Exp Dent 2011; 3: 48790.

11-Lee H, Choi B, Jung J, Zhu S, Lee S, Huh J, et al. Maxillary sinus floor augmentation using autogenous bone grafts and platelet rich fibrin glue with simultaneous implant placement. Oral Surg Oral Med Oral Pathol Oral Radiol Endod 2007; 103: 329-33.

12-Mombelli A, Van Oosten MA, Schürch E Jr, Land NP. The microbiota associated with successful or failing osseointegrated titanium implants. Oral Microbiol Immunol 1987; 2: 145-51.

13-Kasat V, Ladda R. Smoking and dental implants. J Int Soc Prev Community Dent 2012; 2: 38-41.

14-Moy PK. Clinical experience with osseous site development using autogenous bone, bone graft substitutes and membrane barriers. Oral Maxillofac Surg Clin North Am. 2001; 13: 493509.

15-Kumar P, Vinitha B, Fathima G. Bone graft in dentistiry. J Pharm Bioallied Sci. 2013; 5: 125-7.

16-Santagata M, Tozzi U, Prisco RV, Tartaro G, D’Amato S. Autologous bone graft harvested during implant site preparation: histological study. Plast Aesthet Res. 2014;1: 94-7.

17-Thorat M, Pradeep A, Pallavi B. Clinical effect of autologous platelet-rich fibrin in the treatment of intra-bony defects: a controlled clinical trial. J Clin Periodontol. 2011; 38: 925-32.

18-EL Kenawy M H., El Shinnawi UM., Salem A M. and Fakhrdin A H.. Efficacy of platelet rich fibrin (PRF) membrane in immediate dental implant. Mansoura journal of dentistry 2014; 1(3); 78-84.

19- Boora P, Rathee $\mathrm{M}$ and Bhoria M. Effect of platelet rich fibrin (PRF) on peri-implant soft tissue and crestal bone in one stage implant placement: A randomized controlled trial. J Clin Diagn Res. 2015; 9-4. 
20-Huwiler MA, Pjetursson BE, Bosshardt DD, Salvi GE, Lang NP. Resonance Frequency analysis to jawbone characteristics and during early healing of implant installation. Clin Oral Implant Res. 2007; 18: 275-80.

21-Rompen E, DaSilva D, Lundgren AK, Gottlow J, Sennerby L. Stability measurements of a double-threaded titanium implant design with turned or oxidised surface. Appl Osseointegration Res. 2000; 1: 18-20.

22-Qabbani AA, Razak NHA, Kawas SA, Sheikh Abdul Hamid S, Wahbi S, Samsudin AR: The efficacy of immediate implant placement in extraction socket for alveolar bone preservation: A clinical evaluation using three dimensional cone beam computerized tomography and resonance frequency analysis value: J Craniofac Surg, 2017; 28(4): e318-e325. 